\title{
Research
}

\section{Antibiotic treatment of urinary tract infection by community pharmacists:}

\author{
a cross-sectional study
}

\begin{abstract}
Background

Urinary tract infections (UTIs) are one of the most common conditions seen in female patients within primary care. Community pharmacists are familiar with symptomatic UTI management and supplying trimethoprim under patient group direction (PGD) for moderate-to-severe uncomplicated UTIs could improve patient access to treatment.
\end{abstract}

\section{Aim}

To compare the care pathway of patients with UTI symptoms attending GP services with those receiving management, including trimethoprim supply under PGD, via community pharmacies.

\section{Design and setting}

Prospective, cross-sectional, mixed methods approach in 10 community pharmacies within NHS Greater Glasgow and Clyde.

\section{Method}

Pharmacies invited a purposive sample of female patients to participate. Pharmacists had the option of supplying trimethoprim under PGD to patients with moderate-to-severe infection meeting the PGD inclusion criteria. Data from patient lquestionnaires and semi-structured telephone interviews) and pharmacist (questionnaires and semi-structured, face-to-face interviews) were quantitatively and qualitatively analysed.

\section{Results}

Data were recorded on 153 patients, 97 presenting with GP prescriptions and 56 presenting directly in the pharmacy with symptoms suggestive of UTI, of whom 41 received trimethoprim via $\mathrm{PGD}$ and 15 received symptomatic management. Both GP adherence to local infection management guidelines and pharmacist application of PGD inclusion/ exclusion criteria required improvement. There was demand and support, from patients and pharmacists, for access to antibiotic treatments for UTIs, without prescription, through community pharmacies.

\section{Conclusion}

Operating within PGD controls, antibiotic treatments for UTIs could be provided via community pharmacy to improve patient access to treatment which may also maintain antibiotic stewardship and reduce GP workload.

\section{Keywords}

community pharmacy services; health services research; primary health care; trimethoprim; urinary tract infections.

\section{INTRODUCTION}

Urinary tract infections (UTIS) are one of the most common conditions seen in female patients in general practice accounting for $1-3 \%$ of all consultations each year. Diagnosis of UTI is based primarily on signs and symptoms. ${ }^{2}$ If patients present with both dysuria and frequency, the probability of a UTI is greater than $90 \%$, making the diagnosis of UTI (without the need for urinalysis) almost certain. ${ }^{3}$ Symptomatic treatments available over the counter (OTC) from community pharmacies include alkalinising agents, cranberry products, and analgesia. Although urine alkalinisation (for example, with potassium or sodium citratel has traditionally been used to relieve UTI symptoms, there is little evidence to support its use. ${ }^{4,5}$ Antibiotic treatment is frequently unnecessary as uncomplicated UTI is often self-limiting and resolves in a few days without treatment. 6.7 However, in non-pregnant women with moderate-to-severe symptoms, a 3-day course of trimethoprim (200 mg twice daily) or nitrofurantoin (50-100 mg four times daily) is recommended and can shorten the duration of symptoms by 1-2 days. ${ }^{7.8}$

The modern patient-centred NHS is committed to ensuring better, local, faster health care, putting the interests of patients at the centre of reforms thereby offering

JL Booth, MSc, MRPharmS, research pharmacist Pharmaceutical Public Health; DAM Thomson, BSc, FRPharmS, deputy lead - Community Pharmacy Development; SJ Galbraith, BSc prescribing adviser; SM Bryson, MSc, MPH MRPharmS, lead pharmacist, Clinical Governance and Advisory Structures: EM McGovern, MSc, MPH, MRPharmS, lead specialist in Pharmaceutical Public Health, Pharmacy and Prescribing Support Unit, NHS Greater Glasgow and Clyde, Glasgow. AB Mullen, BSc, PhD, PGCE, MRPharmS, FHEA, professor of pharmacy practice Strathclyde Institute of Pharmacy and Biomedical Sciences, University of Strathclyde, Glasgow. C Johnstone, FRCGP, GP, Barony Practice, Paisley. greater choice in how, when, and where to access healthcare services.? Community pharmacies provide convenient access to a qualified healthcare professional without an appointment and routinely advise on minor ailments, including UTIs. As community pharmacists are already familiar with symptomatic UTI management, supplying trimethoprim from community pharmacies under patient group direction (PGD) for uncomplicated UTIs could improve patient access and extend treatment options. ${ }^{10,11} \mathrm{~A}$ $P G D$ is a:

written instruction for the supply or administration of a licensed medicine in an identified clinical situation where the patient may not be individually identified before presenting themselves for treatment."

An NHS Greater Glasgow and Clyde study undertaken in 2010 in 20 community pharmacies identified patient and pharmacist support for improved access to effective treatment from community pharmacies for patients with UTI. ${ }^{12}$ Inappropriate overuse of antibiotics was a concern for participating pharmacists but they supported treatment provision for this indication via a PGD. Despite the supply of trimethoprim under PGD from community pharmacies being novel in Scotland, it is used in some NHS

\section{Address for correspondence}

Elizabeth M McGovern, Pharmaceutical Public Health, Pharmacy and Prescribing Support Unit, West House, Corporate HQ, Gartnavel Royal Hospital, Glasgow, Scotland, G12 OXH.

E-mail: liz.mcgovernaggc.scot.nhs.uk

Submitted: 26 November 2012; Editor's response: 18 December 2012; final acceptance: 16 January 2013

This is the full-length article (published online 25 Mar 2013) of an abridged version published in print. Cite this article as: $\mathbf{B r} \mathbf{J}$ Gen Pract 2013; DOI: 10.3399/bjgp13X665206 


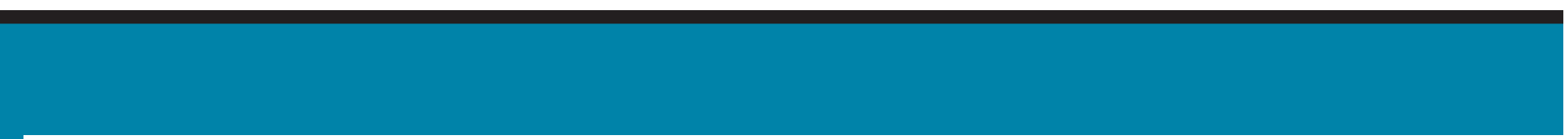

\section{How this fits in}

Evidence for the use of the many symptomatic treatments available from community pharmacies for UTI is unconvincing. There are currently no patient group directions (PGDs) for the supply of trimethoprim via community pharmacies in NHS Scotland and despite their use in community pharmacies in many NHS primary care trusts in England, there are no published studies evaluating PGD use. The study found demand and support, from both patients and pharmacists, for antibiotics to treat UTIs to be available, without prescription, from community pharmacies. Within PGD controls, antibiotic treatments for UTIs could be provided via community pharmacies to improve patient access to treatment, which may also maintain antibiotic stewardship and reduce GP workload.

care trusts (PCTs) in England and Lloyds Pharmacy has recently introduced an online doctor consultation service for the treatment of cystitis. ${ }^{13}$

The study, aligned with NHS Scotland's policy and quality agenda, examined the opportunities for trimethoprim supply under PGD and compared the pathway of patients attending general practice services with those receiving a new model of service provision via community pharmacies. ${ }^{9.14-18}$

METHOD

Setting

NHS Greater Glasgow and Clyde (NHS GGC) is the largest NHS organisation in Scotland and one of the largest in the UK. Covering the city and surrounding urban and rural areas, NHS GGC serves a population of 1.2 million lalmost onequarter of Scotland's population) with 246 general practice surgeries, 30 branch surgeries, and 314 community pharmacies.

\section{Design and analysis}

All 314 community pharmacy contractors were invited to participate in the mixed methods study. A purposive sampling technique was used to select 10 pharmacies, from the 80 positive responses received, to provide maximum variation within the sample of multiple/independent pharmacy status, hours of opening, Scottish Index of Multiple Deprivation Quintile 2009. Urban Rural six-fold classification 2009/2010, and annual prescription and Minor Ailment Scheme volumes. ${ }^{19}$ Based on the information available at the time of selection, the 10 pharmacies were considered a representative sample of both the 80 pharmacies declaring an interest and the total 314 pharmacies within NHS GGC.

The quantitative phase involved staff from the 10 participating community pharmacies recording relevant data (for example, demographics, outcome of consultation and onward referral if appropriate) from eligible patients. Female patients, or representatives reporting symptoms of female patients, were recruited if they presented with symptoms suggestive of UTIs and either:

- requested advice or medication;

- self-selected a product for symptoms suggestive of a UTI; or

- presented with a GP prescription for trimethoprim or nitrofurantoin.

Pharmacists had the option of supplying trimethoprim under PGD to patients with moderate-to-severe infection who met the PGD inclusion criteria (Box 1). Each pharmacy collected data over at least 8 weeks during February to April 2012. Patients, but not their representatives, were invited to complete a short self-completion questionnaire recording their symptoms and attitudes to antibiotics.

The qualitative phase involved interviews with both patients and pharmacists. Patients completing the self-completion questionnaire were also invited to participate in a short semi-structured telephone interview, with the research pharmacist, 4-7 days after consultation in the pharmacy. At the end of the data collection period, face-to-face interviews with pharmacists were conducted using a semi-structured topic guide. Pharmacist interviews were audiorecorded and transcribed verbatim, whereas patient telephone interviews were recorded in a structured format using contemporaneous notes on an interview analysis sheet. All qualitative data were thematically analysed. The research pharmacist coded all interview transcripts which were subsequently reviewed by the principal investigator and an independent, experienced qualitative public health researcher to develop a thematic framework and identify key issues across all participants. ${ }^{20}$

Quantitative data were analysed using SPSS (version 20) to calculate frequencies and $\chi^{2}$ statistics for the relationships between different patient groups (such as general practice or pharmacy model of service provision) for four clinical outcome measures with statistical significance 


\section{Table 1.Patient demographics ( $n=153)$}

\begin{tabular}{lcc} 
Demographics & Frequency & $\%$ \\
\hline Patient age range, years & & \\
$16-40$ & 70 & 46 \\
$41-60$ & 60 & 39 \\
$>60$ & 23 & 15 \\
\hline Who presented & & \\
Patient & 146 & 95 \\
Representative & 7 & 5 \\
\hline Presentation & & \\
GP prescription & 97 & 63 \\
Pharmacy management & 56 & 37 \\
\hline Duration of symptoms, days & & \\
$\leq 2$ & 66 & 43 \\
$3-6$ & 40 & 26 \\
$\geq 7$ & 30 & 20 \\
Missing data & 17 & 11
\end{tabular}

Experienced similar episode previously

Yes $\quad 114 \quad 75$

No

Missing data $\quad 16 \quad 10$

Outcome of consultation

GP prescription

Patient group direction

Symptomatic treatment considered when $P<0.05$ :

- duration of symptoms before seeking help;

- time to symptom resolution after seeking help;

- belief an antibiotic was required for current symptoms; and

- avoidance of GP consultation after receiving pharmacy service.

\section{RESULTS}

\section{Data collection}

During the study period, 153 patients with symptoms suggestive of UTI attended a participating pharmacy (mean 15 patients per pharmacy, range 9-23). Patient demographics are shown in Table 1. A GP prescription was dispensed for 97 patients, $56 \%$ of which were in line with the 3-day treatment recommendation in NHS GGC infection management prescribing guidelines. ${ }^{21}$ Of the 56 patients presenting directly in the pharmacy with symptoms suggestive of UTI, 41 patients received trimethoprim via $P G D$ and 15 patients received symptomatic management. Table 2 shows the symptoms of patients presenting for pharmacy management. Of the 41 patients receiving trimethoprim via PGD, 31 patients (76\%) met the PGD inclusion criteria specified in Box 1 and 29 patients $(71 \%)$ reported potential exclusions (Table 3).

Approximately one-third of all patients presenting in the pharmacy, for management of their symptoms, attended at weekends when general practice surgeries were closed. Patients seeking pharmacy management for their symptoms presented earlier than those with GP prescriptions $\left(\chi^{2}=5.36,1\right.$ degree of freedom [df], $P=0.026$ ). Patients receiving prescriptions ( $n=79,92 \%$ ) were more likely than those receiving pharmacy management ( $n=34,67 \%$ ) to believe an antibiotic was indicated for their current symptoms $\left(\chi^{2}=14.06,1 \mathrm{df}, P<0.001\right)$. Most patients ( $n=80,58 \%$ ) indicated they would be less likely to consult their GP if antibiotics became available from community pharmacies irrespective of whether they received a GP prescription or pharmacy management $\left(\chi^{2}=0.41,1 \mathrm{df}\right.$, $P=0.82$ )

\section{Patient interviews}

Of the 137 patients completing a questionnaire, 94 agreed to be interviewed. Demographics of the 53 patients interviewed are shown in Table 4. Forty-one patients were lost at follow-up for a variety of reasons (Table 5).

Patients were generally satisfied with the delay between symptoms starting and seeking help. In addition they were not always convinced an antibiotic was required and those experiencing similar episodes in the past felt they recognised their symptoms and knew what treatment was required. Almost all patients would preferentially attend the pharmacy for management of their UTI symptoms due to convenience, difficulties obtaining a GP appointment, and reluctance to trouble GPs with a non-emergency problem:

I'm working all the time and can't get an appointment at doctor. Appointments are only during the daytime but that pharmacy is open at night so / went there after work. ' (patient supplied trimethoprim via PGD)

'Felt bad making an urgent appointment with my doctor but if available from the pharmacy would have saved a doctor appointment.' (patient presented with GP prescription]

more willing to use service from pharmacy than waste valuable doctor time.' lpatient presented with GP prescription)

Some patients were aware they had an infection but did not believe an antibiotic was required:

'To be honest didn't think I needed an antibiotic. Just thought here we are again I need to take the medicine and it'll go away.' (patient supplied with symptomatic relief)

Four of the interviewees who received pharmacy management intended to consult their GP due to either persistent symptoms (three patients) or vaginal candidiasis (one patient). There was no significant difference in time to resolution of symptoms between those receiving a GP prescription or pharmacy management $\left(\chi^{2}=0.41,1 \mathrm{df}, P=0.52\right)$.

\section{Pharmacist interviews}

The 10 pharmacists interviewed were unanimously in favour of providing management from the pharmacy, valuing the increased professional role. There was discomfort among pharmacists when supplying alkalinising agents. There is limited evidence of efficacy for these agents and supplying trimethoprim 

Table 3. Presenting symptoms of patients supplied trimethoprim
under patient group direction $(n=41)$

\begin{tabular}{lccl} 
Symptoms & Frequency & $\%$ & Comment \\
\hline Presenting symptoms & a & & \\
Dysuria & 36 & 88 & - \\
Frequency/urgency & 36 & 88 & Potential exclusion \\
Back/abdominal pain & 20 & 49 & Potential exclusion \\
Fever/chills & 8 & 20 & - \\
Haematuria & 2 & 5 & Potential exclusion \\
Vaginal discharge & 1 & 2 & - \\
Other (for example, malaise) & 0 & - & $17 / 21$ met PGD inclusion criteria \\
Number of symptoms & & & $14 / 17$ met PGD inclusion criteria \\
$\geq 3$ & 21 & 51 & - \\
2 & 17 & 42 & - \\
1 & 3 & 7 & - \\
\hline Duration of symptoms, days & & & - \\
$\leq 2$ & 22 & 54 & - \\
$3-6$ & 15 & 36 & - \\
$\geq 7$ & 4 & 10 & \\
\hline Day of presentation & & & \\
Monday-Thursday & 14 & 34 & \\
Friday/Saturday/Sunday & 27 & 66 & \\
\hline
\end{tabular}

${ }^{a}$ Numbers do not add up to $100 \%$ as patients could present with several symptoms.

\section{Table 4. Demographics of interviewed patients ( $n=53$ )}

\begin{tabular}{lcc} 
Demographics & Frequency & $\%$ \\
\hline Age, years & 24 & 45 \\
$16-40$ & 22 & 42 \\
$41-60$ & 7 & 13 \\
$>60$ & 1 & 2 \\
\hline Time since presentation in pharmacy & \\
$<4$ days & 46 & 87 \\
$4-7$ days & 6 & 11 \\
$>7$ days & 30 & 57 \\
Outcome of consultation & 18 & 34 \\
GP prescription & 5 & 9 \\
Supply under PGD & & \\
Symptomatic treatment & 5 & 49 \\
\hline Time to resolution of symptoms, days & \\
$\leq 2$ & 26 & 34 \\
$3-5$ & 18 & 2 \\
6-7 & 1 & 15 \\
Unresolved at interview & 8 & \\
\hline PGD = patient group direction. & & \\
& &
\end{tabular}

Table 5. Reasons for interviews not being undertaken $(n=41)$

\begin{tabular}{lc} 
Reason & $\boldsymbol{n}(\mathbf{\%})$ \\
\hline Patient unavailable & $21(51)$ \\
\hline Time of interview unsuitable & $5(13)$ \\
\hline Patient unwell & $1(2)$ \\
\hline Patient unsuitable & $1(2)$ \\
\hline Patient unwilling & $2(5)$ \\
\hline Time delay in receiving details & $11(27)$ \\
\hline
\end{tabular}

offers appropriate patients an effective alternative:

you feel guilty giving the sachets that you know aren't going to work.'

if it [PGD] was rolled out it would be a great addition to the ... pharmacy armoury.

Pharmacists recognised the need for antibiotic stewardship and welcomed following the strict PGD protocol with clear inclusion/exclusion criteria for trimethoprim supply. Some concern was expressed regarding potential patient misuse and over-demand:

it's quite a serious issue giving out antibiotics without a prescription ...

I think a lot of the time they just assumed they were going to get an antibiotic before I had even asked them the questions or seen if it was suitable. And ... sometimes I found that when I was speaking to the patients ... they just gave me all their symptoms because they thought I better tell them everything so / get an antibiotic

I had one woman that said she had discharge and then I said to her "look it is really important that you should go to your doctor" and then straight away she went "well I don't really have a discharge ... I kind of had a discharge, I get that anyway, it wasn't discoloured or anything, it is just normal".

Almost universally pharmacists suggested a model mirrored on the existing Emergency Hormonal Contraception service as the preferred model for potential wider roll-out, as part of the national pharmacy contract. $^{22}$

\section{DISCUSSION}

\section{Summary}

Evidence to support the use of symptomatic treatments currently available from community pharmacies is unconvincing. ${ }^{4}$ The current study provides evidence of demand and support, from patients and pharmacists, for improved access to treatment for UTIs through community pharmacies. Participating pharmacists were keen for a community pharmacy service managing UTI symptoms using a trimethoprim PGD to be introduced either as a local or national scheme using a similar arrangement modelled on the existing emergency hormonal contraception service. ${ }^{22}$

The potential pharmacist variance to the PGD exclusion/inclusion criteria need to be considered and is most likely the result of ambiguity in the data collection instrument rather than non-adherence to PGD criteria; that is, the list of possible symptoms on the pharmacist questionnaire included 'back/abdominal pain' as one single criteria, whereas PGD criteria were explicit and included 'suprapubic tenderness' as an inclusion and 'loin pain/tenderness' as an exclusion. Alternatively the potential variance to the PGD inclusion/exclusion criteria may reflect a discrepancy between patients' selfreported symptoms and the pharmacist's professional assessment. These symptoms would be discussed, as part of the routine clinical dialogue with the patient, to guide the assessment of appropriateness of trimethoprim supply and may reflect differences in interpretation of presenting symptoms or professional judgement on the consequences of non-supply.

If pharmacists' professional responsibility to practice evidence-based principles conflict with patient need then potential causes for this discrepancy should be addressed in further research. Similarly the low adherence to recommended 3-day duration of prescribed therapy may reflect GP assessment of a more complicated UTI which cannot be determined from data recorded. Nevertheless a health boardwide education initiative to improve current poor adherence to local antimicrobial infection management guidelines may be required and should focus jointly on pharmacists and GPs. ${ }^{23}$ Operating within 
PGD controls, antibiotics for UTIs could be provided via community pharmacy to improve patient access to treatment, which may also maintain antibiotic stewardship and reduce GP workload.

\section{Strengths and limitations}

Although community pharmacists are familiar with PGDs to supply other prescription-only medicines, there are no PGDs for the supply of trimethoprim in current regular use within NHS Scotland community pharmacies. This novel study provides evidence for a new model of care in an under-researched area.

The limitations of a small sample size and limited clinical information is recognised. In addition, there is currently no patient expectation to receive antibiotics from pharmacies and, with time, patterns of presenting behaviour may change as patients become increasingly aware pharmacies can provide the service.

Patient interviews were recorded using contemporaneous notes, which may have introduced researcher bias. The researcher is a pharmacist whose own professional opinions may have influenced recall/interpretation of patients' views and experiences.

\section{Comparison with existing literature}

As this study was novel, drawing suitable comparators from the existing literature is limited. In addition, despite PGDs for the supply of trimethoprim from community pharmacies being used in many NHS PCTs in England, there are no published studies researching UTI management in community pharmacies.

In general practice in NHS England, trimethoprim prescriptions for 3-days treatment rose from 5\% in 1998 to $18 \%$ in 2001 but in 2008 still remained below $30 \% .^{1}$ Although results from this study demonstrate further improvement, there is no room for complacency as inappropriate treatment duration is an antibiotic stewardship issue. ${ }^{24}$

\section{Implications for practice and research}

The feasibility of introducing a local arrangement or national scheme for a community pharmacy-led service managing UTI symptoms with wider availability of trimethoprim via PGD should be investigated. Current adherence to infection management guidelines for UTI was poor and efforts are needed to further encourage GP adherence to current prescribing guidelines, especially to duration of treatment. Despite participating pharmacists being aware of the need to supply the antibiotic with caution, adherence to the PGD inclusion-exclusion criteria required improvement. Further research is required to investigate the underlying causes of pharmacist behaviours when empowered in an antibiotic stewardship role.

The West of Scotland Research and Ethics Committee (REC) considered the study to be service development/evaluation and, therefore, deemed REC approval unnecessary. The NHS GGC Clinical Governance Support Unit was subsequently informed of the study.

\section{Provenance}

Freely submitted; externally peer reviewed.

\section{Competing interests}

The authors have declared no competing interests.

\section{Acknowledgements}

With thanks to PTECO for funding the study and to the 10 pharmacies, their pharmacists, pharmacy support staff and patients for participating in the study. Thanks to Rona Dougall and Susan Fleming, NHS GGC Public Health Researchers, for their help and advice and Dawn MacCloy, Administrator, NHS GGC Pharmaceutical Public Health, for designing the study database.

\section{Discuss this article}

Contribute and read comments about this article on the Discussion Forum: http://www.rcgp.org.uk/bjgp-discuss 


\section{REFERENCES}

1. National Institute for Health and Clinical Excellence. Common infections: urinary tract infection. Data focused commentary: management of acute uncomplicated urinary tract infection. London: NICE, 2009. http://wnw.npc.nhs.uk/therapeutics/ common_infections/uti/resources/dfc_common_infect_uti.pdf laccessed 25 Feb 2013).

2. Scottish Intercollegiate Guidelines Network. Management of suspected bacterial urinary tract infection (SIGN 88). Scotland: Health Improvement Scotland, 2012.

3. Medicines Resource Centre. Acute uncomplicated urinary tract infection in women. MeReC Bulletin 2006; 17(3): 18-20.

4. Munday PE, Savage S. Cymalon in the management of urinary tract symptoms. Genitourin Med 1990; 66(6): 461.

5. Spooner JB. Alkalinisation in the management of cystitis. J Intern Med Res 1984; 12(1): 30-34

6. Balakrishnan I, Hill V. Dealing with urinary tract infections. The Pharmaceutical Journal 2010; 287: 687-690.

7. Little $\mathrm{P}$, Turner S, Rumsby K, et al. Dipsticks and diagnostic algorithms in urinary tract infection: development and validation, randomised trial, economic analysis, observational cohort and qualitative study. Health Technol Assess 2009; 13(19): iii-iv, ix-xi, 1-73.

8. Milo G, Katchman E, Paul M, et al. Duration of antibacterial treatment for uncomplicated urinary tract infection in women. Cochrane Database Syst Rev 2005; 2: CD004682. DOI: 10.1002/14651858.CD004682.pub2.

9. Scottish Government. Better health, better care. Edinburgh: NHS Scotland, 2007.

10. National Prescribing Centre. Patient group directions - a practical guide and framework of competencies for all professionals using patient group directions. England: National Prescribing Centre, 2004.

11. Bussey A, Rutter T. 'To PGD or not to PGD' - that is not the only question. Pharmacy Management 2009; 25(1): 3-7.

12. Booth J. Management of urinary tract infection symptoms in patients attending community pharmacies. NHS Greater Glasgow and Clyde: Pharmaceutical Public Health, July 2010.

13. Lloyds Pharmacy. Online doctor. Cystitis treatment. https://onlinedoctor. lloydspharmacy.com/womens-health/cystitis/cystitis-treatment laccessed 25 Feb 2013).
14. Scottish Government. Role of pharmacists to be reviewed. Edinburgh: NHS Scotland, 2011.http://uww.scotland.gov.uk/News/Releases/2011/10/17101158 (accessed 25 Feb 2013).

15. NHS Scotland. Shifting the balance of care. Edinburgh: NHS Scotland. http:// unw.shiftingthebalance.scot.nhs.uk/ laccessed 25 Feb 2013).

16. NHS Scotland. Informing, engaging and consulting people in developing health and community care services. Edinburgh: NHS Scotland, 2004. http://www. clinicalgovernance.scot.nhs.uk/documents/IECGuidance.pdf laccessed 25 Feb 2013).

17. Scottish Executive Health Department. Research governance framework for health and community care. 2nd edn. Edinburgh: Scottish Executive Health Department, 2006.

18. Scottish Government. The healthcare quality strategy for NHS Scotland. Edinburgh: Scottish Government, 2010.

19. Community Pharmacy Scotland. NHS care services: minor ailment scheme. Edinburgh: Community Pharmacy Scotland. http://www. communitypharmacyscotland.org.uk/nhs_care_services/minor_ailment_ service/minor_ailment_service.asp laccessed 25 Feb 2013).

20. National Centre for Social Research. The framework method for qualitative data analysis. London: NatCen Social Research. http://www.natcen.ac.uk/ourexpertise/framework laccessed 25 Feb 2013).

21. NHS Greater Glasgow and Clyde. Infection management in adults: guidance for primary care. NHS Greater Glasgow and Clyde: Antimicrobial Management Team, 2009.

22. Community Pharmacy Scotland. NHS Care Services: Public health services sexual health specifications. Edinburgh: Community Pharmacy Scotland. http:// www.communitypharmacyscotland.org.uk/nhs_care_services/public_health_ service/phs_sexual_health_specifications.asp (accessed 25 Feb 2013).

23. Royal College of General Practitioners Scotland and Royal Pharmaceutical Society in Scotland. Joint statement: breaking down the barriers - how pharmacists and GPS can work together to improve patient care. Edinburgh: RCGP Scotland and RPS Scotland. http://www.rpharms.com/currentcampaigns--scotland-pdfs/rpsscotlandrcgpjointstatement.pdf laccessed 25 Feb 2013).

24. Dryden MS, Cooke J, Davey P. Antibiotic stewardship - more education and regulation not more availability? J Antimicrob Chemother 2009; 64(5): 885-888. 